\title{
ЭКСПЕРИМЕНТАЛЬНЫЕ ПОДХОДЫ К ТАРГЕТНОМУ РЕДАКТИРОВАНИЮ ГЕНА CFTR С ПОМОЩЬЮ CRISPR-CAS9
}

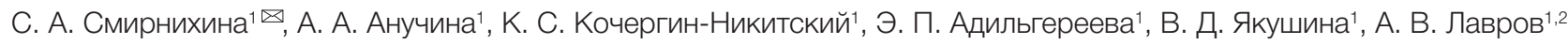

1 Лаборатория мутагенеза, Медико-генетический научный центр, Москва

${ }^{2}$ Кафедра молекулярной и клеточной генетики, медико-биологический факультет,

Российский национальный исследовательский медицинский университет имени Н. И. Пирогова, Москва

\begin{abstract}
Муковисцидоз - тяжелое аутосомно-рецессивное заболевание, обусловленное мутациями в гене CFTR, основной из которых в европейской популяции является F508del. Патогенетическая терапия существенно улучшила прогноз для жизни у пациентов с муковисцидозом, однако генная терапия не оказалась такой эффективной, как ожидалось. Геномное редактирование, в том числе с помощью CRISPR-Cas9, открывает новые возможности для этиотропного лечения, так как позволяет исправить мутации в клетках. Целью исследования было сравнение эффективности коррекции мутации F508del с помощью различных комбинаций направляющих PHК и Cas9 и повышение эфффективности редактирования. Работу проводили на культуре клеток НЕК293Т, эффективность редактирования генома оценивали с помощью анализа T7E1, как на геномном, так и на плазмидном сайтах. Наиболее эффективной оказалась комбинация SaCas9 вместе c PHK на мутацию F508del - произошло редактирование 29\% аллелей. Комбинация аналогичной направляющей PHK на F508del для SpCas9 показала небольшую эффективность редактирования, что связано с низкой экспрессией направляющей РНК. Были предприняты попытки увеличения экспрессии данной РНК с помощью разных подходов, однако повышения эффективности ее работы получено не было. Стабилизация направляющей PHK путем добавления в последовательность G-квадруплекса, укорочения и добавления GG в 5'-область также не принесла результатов. Вероятно, низкая эффективность работы использованной направляющей РНК обусловлена ее нуклеотидной последовательностью, что ограничивает ее использование.
\end{abstract}

Ключевые слова: муковисцидоз, CRISPR-Cas9, CFTR, геномное редактирование, мутация F508del, направляющие PHK Финансирование: раздел «Редактирование CFTR локуса» поддержан грантом РНФ (Соглашение № 17-75-20095), разделы «Увеличение экспрессии направляющих PHK» и «Увеличение эффеективности редактирования CFTR локуса» поддержаны Российской академией наук и государственным заданием ФГБНУ «МГНЦ».

$\bowtie$ Для корреспонденции: Светлана Анатольевна Смирнихина ул. Москворечье, д. 1, г. Москва, 115522; smirnikhinas@gmail.com Статья получена: 15.03.2018 Статья принята к печати: 20.03.2018 DOI: $10.24075 /$ vrgmu.2018.022

\section{EXPERIMENTAL APPROACHES TO THE TARGET EDITING OF THE CFTR GENE USING CRISPR-CAS9}

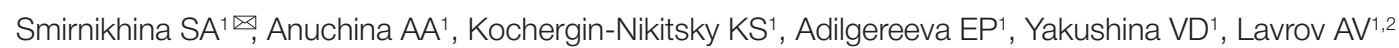

${ }^{1}$ Laboratory of Mutagenesis, Research Centre for Medical Genetics, Moscow

2Department of Molecular and Cellular Genetics,

Pirogov Russian National Research Medical University, Moscow

Cystic fibrosis is a severe autosomal recessive disease caused by mutations in the CFTR gene. The most common CFTR mutation occurring in the European population is F508del. Advances in the management of patients with cystic fibrosis aimed at blocking disease progression have considerably improved the prognosis, but gene therapy has turned to be less effective than expected. Capable of correcting mutations direct in the cells, genome editing, and specifically the CRISPR-Cas9 technology, raises hope of causal treatment for patients with cystic fibrosis. The aim of this work was to compare and improve the efficacy of F508del editing using different combinations of guide RNAs and Cas9. The study was carried out in HEK293T cells. The efficacy of editing was assessed for both plasmid and genomic sites by T7E1 analysis. The best effect was demonstrated by a combination of SaCas9 and sgRNA targeting F508del: 29\% of alleles were successfully edited. A combination of SpCas9 and a similar sgRNA showed low efficacy due to the low expression of this guide RNA. All attempts to improve its expression failed. SgRNA stabilization by introducing a G-quadruplex into the sgRNA sequence and adding GG to the $5^{\prime}$-region also did not work. Perhaps, low performance of this guide RNA is determined by its nucleotide sequence, limiting its use.

Keywords: cystic fibrosis, CRISPR-Cas9, CFTR, genome editing, F508del mutation, guide RNA

Funding: the section Editing of the CFTR locus was supported by the grant of the Russian Science Foundation (Agreement 17-75-20095), the sections Increasing the expression of guide RNAs and Improving the efficacy of CFTR locus editing were supported by the Russian Academy of Sciences and the state assignment of FASO Russia.

Correspondence should be addressed: Svetlana Smirnikhina Moskvorechie 1, Moscow, 115522; smirnikhinas@gmail.com

Received: 15.03.2018 Accepted: 20.03.2018

DOI: 10.24075/brsmu.2018.022 
Муковисцидоз (МВ, ОМІМ\#219700) - аутосомнорецессивное заболевание, обусловленное мутациями в гене CFTR (cystic fibrosis transmembrane conductance regulator), приводящими к нарушению транспорта ионов хлора и натрия через клеточную мембрану. Это одно из самых распространенных наследственных заболеваний с частотой встречаемости 1 на 4500 человек, при этом частота носительства мутаций гена CFTR достигает 1 на 25 человек [1]. Основные клинические симптомы связаны с поражением легких, являющимся основной причиной смерти пациентов [2], однако могут также вовлекаться поджелудочная железа, печень, кишечник. Самой частой мутацией в гене CFTR является F508del, приводящая к нарушению созревания белка CFTR и его полному отсутствию на поверхности клеток [3]. Патогенетическая терапия МВ в последние десятилетия достигла существенных результатов [4-7], однако этиотропного лечения до сих пор не существует.

Технологии геномного редактирования, включая использование CRISPR-Cas9, позволяют по-новому взглянуть на возможности генной терапии наследственных заболеваний [8]. Их можно использовать для коррекции («редактирования») мутаций и разрабатывать этиотропную терапию неизлечимых до сих пор болезней [9-12]. Опубликованные ранее работы по исправлению мутации F508del с помощью различных методов геномного редактирования [13-20] позволяют на их основе развивать и совершенствовать новые подходы к редактированию F508del. Существенный недостаток опубликованных работ - крайне низкая эффективность успешного редактирования (<<1\% клеток), что является общей проблемой технологии геномного редактирования.

Для разработки более эффективного способа коррекции мутации F508del мы подобрали несколько направляющих PHK на место вокруг мутации и использовали разные ранее не использованные для этих целей ортологи Cas9, создав таким образом несколько комбинаций Cas9 + sgPHK для определения наиболее эфффективной.

Целью работы было сравнение эфрфективности способов коррекции мутации F508del с помощью различных комбинаций направляющих PHK и Cas9 и повышение эффективности редактирования.

\section{МАТЕРИАЛЫ И МЕТОДЫ}

Исходные плазмиды для CRISPR-Cas9 были любезно предоставлены Feng Zhang (Addgene \#71814 и \#61591) и Keith Joung (Addgene \#72249). Направляющие PHK (sgRNA) для SpCas9, SpCas9(HF4) и SaCas9 подобрали с помощью свободного программного обеспечения, разработанного Broad Institute (США) (http://portals. broadinstitute.org/gpp/public/analysis-tools/sgrna-design). В результате клонирования получили плазмиды (рис. 1). Для проверки эффективности полученных систем локус вокруг мутации F508del в 400 п.о. клонировали в плазмиду pGEM-TA-CFTR, которую котрансфицировали с плазмидой, экспрессирующей Cas9 и sgRNA. Клеточную культуру НЕК293Т (любезно предоставлена к. б. н. М. Ю. Скобловым, лаборатория функциональной геномики ФГБНУ «МГНЦ", Москва), культивировали в DMEM (ПанЭко, Россия) с добавлением 10\% эмбриональной бычьей сыворотки (PAA Laboratories GmbH, Австрия), 100 U/мл/100 мкг/мл пенициллин/стрептомицина и 4 мM L-глутамина (ПанЭко, Россия). Для проверки роли температуры культивирования клетки после трансфекции культивировали в двух условиях: стандартных и при пониженной температуре. По стандартной методике культивировали 72 ч при $37^{\circ} \mathrm{C}$; при втором способе клетки сначала культивировали 24 ч при $37^{\circ} \mathrm{C}$, затем 48 ч при $30{ }^{\circ} \mathrm{C}$. Кальций-фоосфатную трансфекцию клеток НЕК293Т проводили в 12-луночных планшетах при 50\% конфлуентности, как описано ранее [21], суммарное количество плазмид на лунку составляло 1,5 или 5,5 мкг (при котрансфекции 1 или 5 мкг плазмиды с Cas9 и sgRNA и 0,5 мкг целевой плазмиды). Через 6 ч после транссекции среду меняли на полную ростовую, содержащую 10\% эмбриональной бычьей сыворотки. В качестве репортерной плазмиды использовали pEGFP-C1 (Clontech, США). Выделение ДНК проводили с помощью набора Genomic DNA-Tissue MiniPrep (ZymoResearch, СШA), согласно протоколу производителя. Т7Е1-анализ проводили, как описано ранее [22]: продукты амплификации фрагмента ДНК с предположительными вставками-делециями на месте двухцепочечного разреза подвергали нагреву и резкому охлаждению, образование гетеродуплексов фиксировали

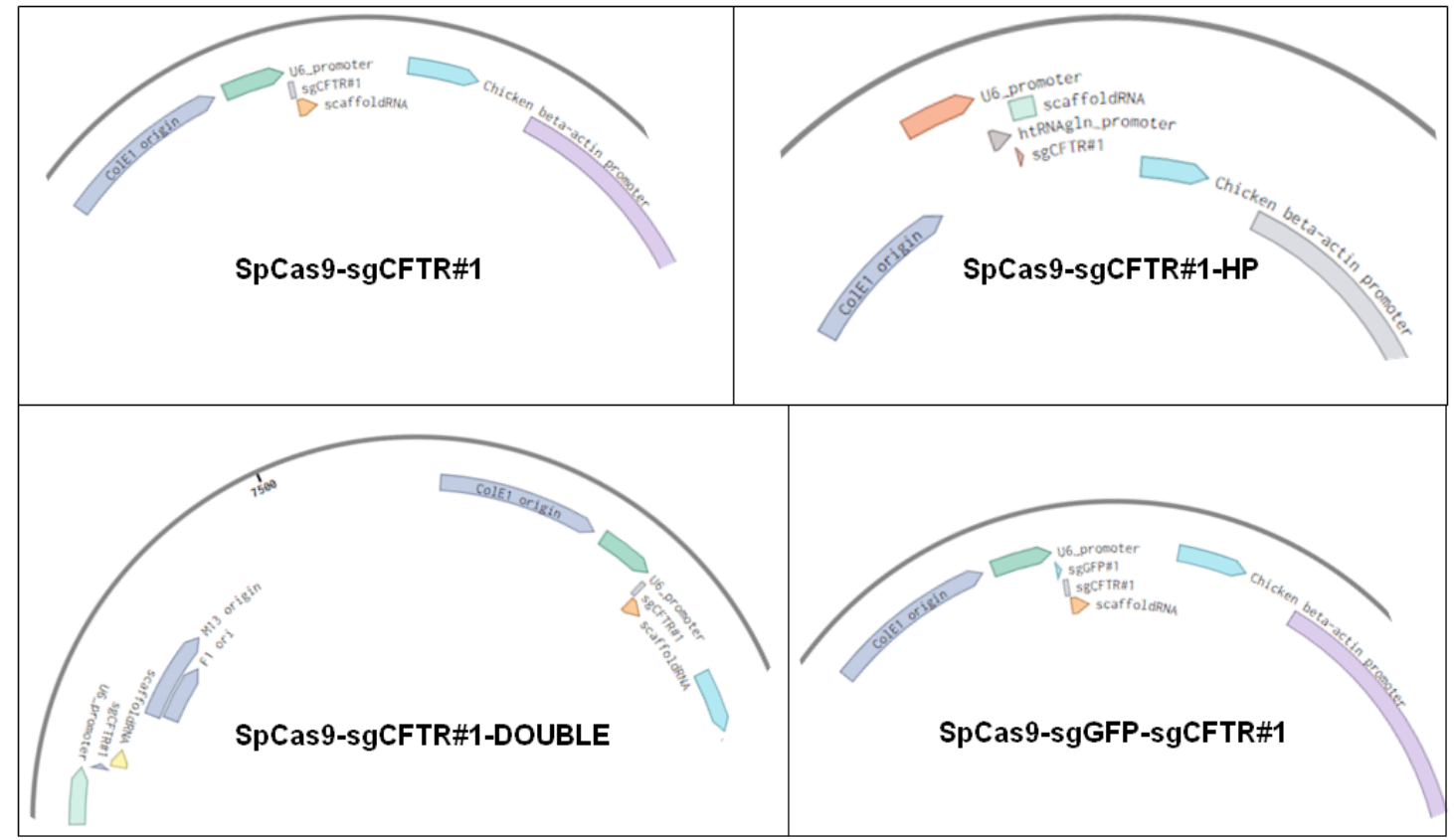

Рис. 1. Карты полученных плазмид для геномного редактирования локуса CFTR мутации F508del 
по наличию дополнительных полос при электрофорезе после обработки гетеродуплексов эндонуклеазой T7E1.

\section{РЕЗУЛЬТАТЫ ИССЛЕДОВАНИЯ}

\section{Редактирование CFTR-локуса}

В работе проводили сравнение эффективности геномного редактирования мутации F508del в гене CFTR с использованием двух мутировавших SpCas9 (eSpCas9(1.1) [23] и SpCas9(HF4) [24]) и SaCas9 [25] с разными sgRNA в области мутации. Для SpCas9 подобрали три sgRNA на последовательность 10 экзона гена CFTR в месте мутации F508del (рис. 2). При этом РАМ-последовательность для sgCFTR\#1 появлялась только при наличии мутации F508del. Вторую sgRNA (sgCFTR\#2) подобрали на последовательность ДНК рядом с мутацией, с ее помощью можно редактировать как последовательность с мутацией, так и дикий тип. Третью sgRNA (sgCFTR\#3) подобрали на последовательность, находящуюся в 85 нуклеотидах от мутации [13]. SaCas9 использует в своей работе другой РАМ, вследствие чего для этой нуклеазы подобрали отдельную sgRNA (saCFTR\#3) - непосредственно на мутацию. Из-за того что в клеточной культуре НЕК293Т отсутствует мутация F508del и организация геномного сайта предположительно может влиять на эффективность редактирования, sgRNA тестировали также на специально созданной плазмиде с локусом вокруг мутации F508del, которую котрансфицировали с плазмидой, экспрессирующей Cas9 и sgRNA.

Наиболее эффективной комбинацией Cas9 и sgRNA оказалась комбинация SaCas9-saCFTR\#3 - произошло редактирование 29\% аллелей (рис. 3). SgCFTR\#1 в комбинации с разными SpCas9 продемонстрировала в среднем эффективность редактирования, равную 13\%. SgCFTR\#2 показала эффективность редактирования в среднем 18\% (16\% на плазмидном сайте и 22\% - на геномном), sgCFTR\#3 - в среднем 12\% (6\% на плазмидном сайте и $14 \%$ - на геномном). Активность редактирования с использованием sgCFTR\#1 сопоставима или ниже активности других направляющих РНК, в том числе контрольной sgGFP, подобранной на ген EGFP (рис. 3).

\section{Увеличение экспрессии направляющих РНК}

Проведенная ранее работа показала, что невысокая эффективность редактирования с помощью sgCFTR\#1 коррелирует с низким уровнем экспрессии этой sgRNA [22]. С целью увеличения экспрессии sgCFTR\#1 в плазмиду добавили дополнительную кассету промотор + sgCFTR\#1 (SpCas9-sgCFTR\#1-DOUBLE), однако она не оказала существенного влияния на эффективность работы этой направляющей PHK (рис. 4). В работе использовали высокоэффективную sgRNA, подобранную к гену EGFP (sgGFP), в качестве положительного контроля. Так как sgGFP всегда активно экспрессировалась и показывала высокую эффективность, мы соединили две sgRNA, т. е. sgGFP и sgCFTR\#1 (SpCas9-sgGFP-sgCFTR\#1), однако слитная sgRNA привела K снижению эффективности редактирования локуса CFTR (рис. 4).

В ряде работ показано, что экспрессию можно увеличить с помощью гибридного промотора, т. е. состоящего из двух слитных промоторов [26]. Вероятный механизм действия - привлечение разными промоторами разных транскрипционных факторов. В используемых нами плазмидах sgRNA экспрессируется с промотора U6, который является стандартным для CRISPR-Cas9. Однако в ряде работ показано, что экспрессия sgRNA с промотора tRNAgln выше [26, 27]. Поэтому в работе решено было клонировать в плазмиду гибридный промотор, состоящий из промоторов U6 и tRNAgln (добавление HP после названия плазмиды). Как видно на рис. 5, эффективность работы всех sgRNA снизилась, за исключением sgCFTR\#1, чья активность незначительно увеличилась.

\section{TCAGAGGGTAAAATTAAGCACAGTGGAAGAATTTCATTCTGTTCTCAGTTTTCCTGGATTATGCCTGGCACCATTAAAGAAAATATCA ET GGTGTITCCTATGATGAATATAGATACAGAAGCGT AGTCTCCCATTTTAATTCGTGTCACCTTCTTAAAGTAAGACAAGAGTCAAAGGACCTAATACGGACCGTGGTAATTTCTITTATAGT FA- CCACAAAGGATACTACTTATATCTATGTCTTCGCAA

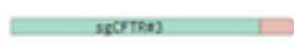

Рис. 2. Используемые в работе sgRNA на локус CFTR

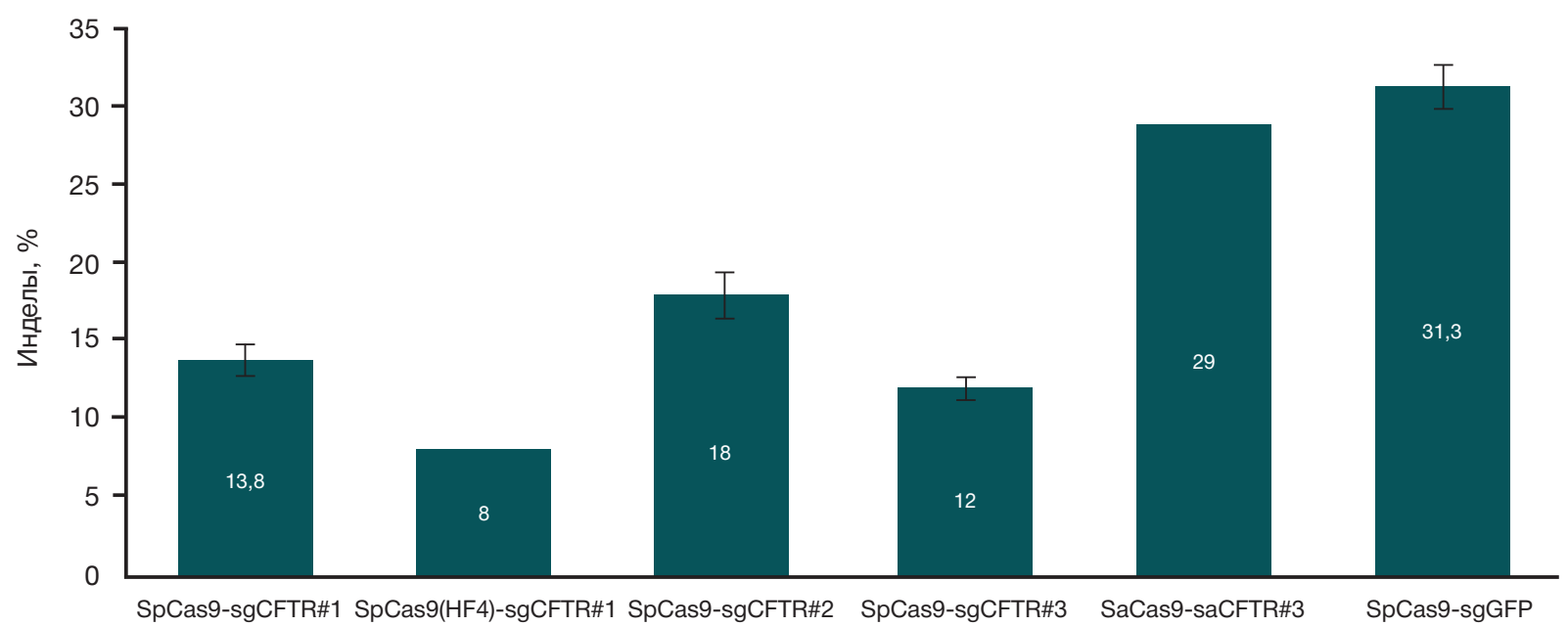

Рис. 3. Сравнение эффективности редактирования CFTR и EGFP в культуре НЕК293Т через 48-72 ч после трансфекции. Данные представлены как среднее значение со стандартной ошибкой среднего 


\section{Увеличение эффективности редактирования CFTR-локуса}

Известно, что молекулы sgRNA размером короче 20 нуклеотидов и начинающиеся на G- или GG-нуклеотид более эффективны [28]. SgCFTR\#2 и sgCFTR\#3 содержали в 5'-области два нуклеотида GG, поэтому в работе укоротили молекулу sgRNA с 5'-конца до 17 нуклеотидов (SpCas9-sgCFTR\#2(GG17) и SpCas9-sgCFTR\#3(GG17) соответственно). SgCFTR\#1 не содержала в 5'-области GG, поэтому ее укоротили до 19 нуклеотидов и заменили начальные нуклеотиды CC на GG (SpCas9sgCFTR\#1(gg19)). Модификации sgCFTR\#1 и sgCFTR\#3 привели к уменьшению их активности с 20,3 и 11,8 до 8,7\% и 0\%, соответственно (рис. 6), тогда как модификация sgCFTR\#2 увеличила ее эффективность с 10,5 до 22,1\%.

Предполагая, что эффективность работы направляющих РНК связана с их стабильностью, к последовательностям sgCFTR\#1 и sgGFP с 5'-конца добавили последовательность CACCGGGAGGGCGGGGAGGG, чтобы создать условия для образования G-квадруплексов sgCFTR\#1quad и sgGFPquad соответственно, повышающих стабильность sgRNA [29]. Результаты работы показали, что эффективность разрезания таргетной ДНК с использованием измененных направляющих PHК уменьшилась из-за снижения их экспрессии в 2-16 раз относительно немодифицированных sgRNA [22].

Наконец, осуществляли попытки стабилизации нуклеазы SpCas9 с помощью временного культивирования трансфицированных клеток при $30{ }^{\circ} \mathrm{C}[14,30]$. Эфффективность редактирования CFTR в результате этого эксперимента снизилась почти в 2 раза - с 17,6 до 10,9\% (табл.).

\section{ОБСУЖДЕНИЕ РЕЗУЛЬТАТОВ}

Попытки коррекции мутаций в гене CFTR с помощью геномного редактирования начались в 2012 г. [14], однако до сих пор нет готового эффективного решения. Связано это, прежде всего, с низкой эффективностью данных подходов. Небольшой процент клеток с исправленной мутацией заставляет использовать клеточную селекцию $[13,16]$, что существенно увеличивает себестоимость методики и удлиняет процесс получения необходимой клеточной культуры. Кроме того, такой подход не позволяет лечить заболевание системно.

Развивающиеся технологии геномного редактирования позволяют усовершенствовать систему, увеличивая ее эффективность и специфичность. В своей работе

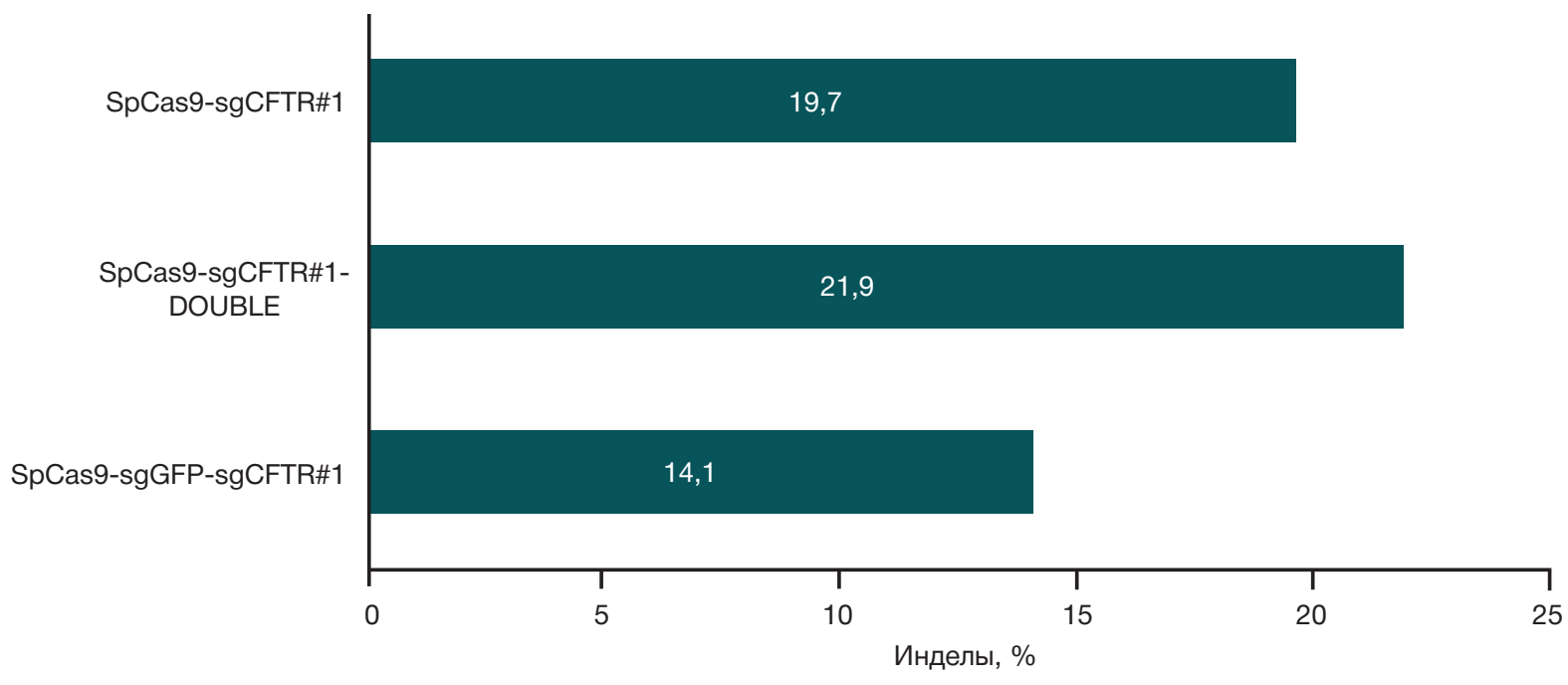

Рис. 4. Сравнение эффективности редактирования CFTR в культуре НЕК293Т. Данные представлены как среднее значение

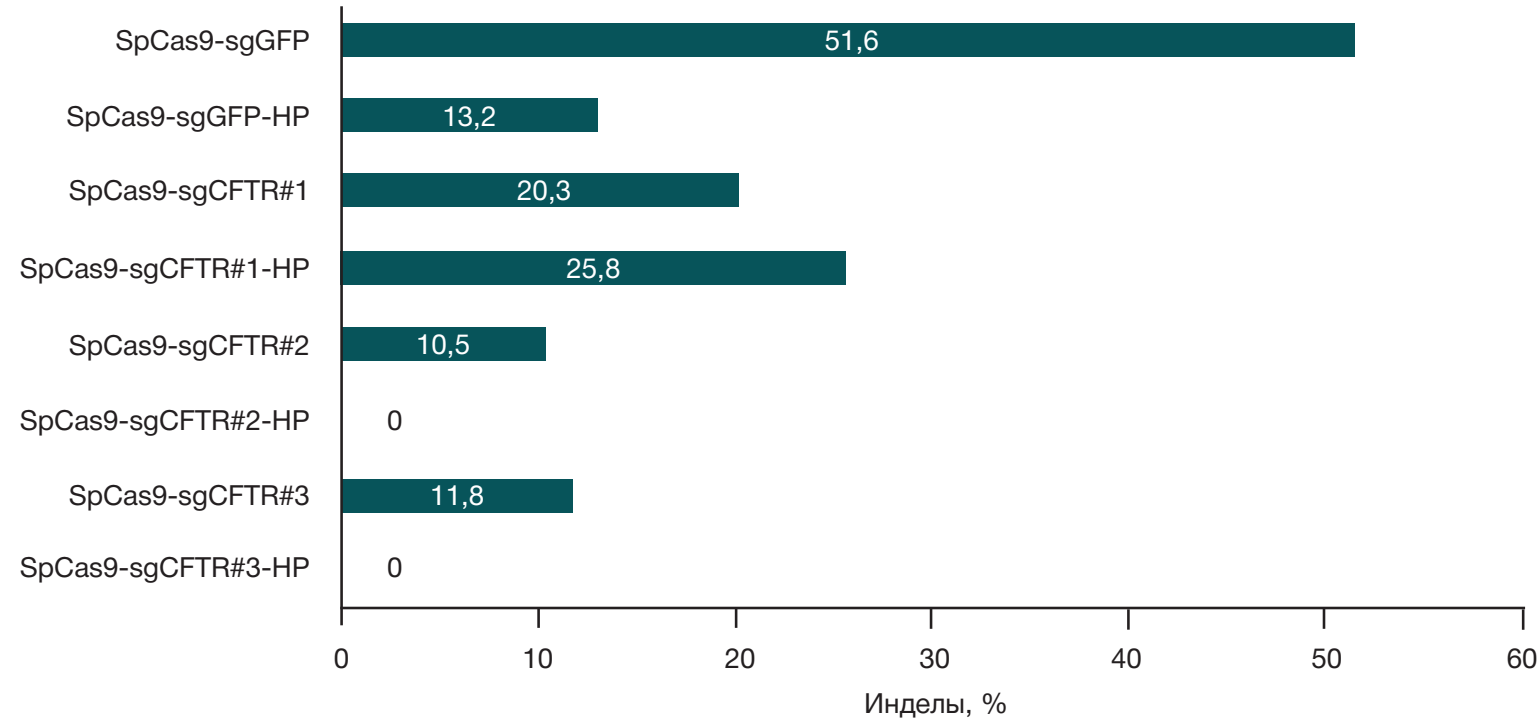

Рис. 5. Сравнение эффективности редактирования CFTR и EGFP в культуре HEK293Т с использованием sgRNA, экспрессирующихся со стандартного промотора U6 и с гибридного промотора U6-tRNAgln (плазмиды HP). Данные представлены как среднее значение 
мы используем более специфичные ферменты для редактирования генома [23, 24], что повышает безопасность методики. Кроме того, мы разрабатываем метод редактирования, работающий только на мутировавшем сайте, используя действие направляющих РНК непосредственно на мутацию F508del. Это открывает новые возможности: должно происходить редактирование не выделенных клеток, а в организме, так как меняться будут только аллели с мутацией. К тому же, новый подход позволит избежать повторного разрезания уже отредактированного локуса.

Однако в ходе работы было показано, что эффективность редактирования с помощью sgCFTR\#1, подобранной на мутацию F508del, ниже по сравнению с большинством используемых нами sgRNA. Основной причиной низкой эффективности стала низкая экспрессия sgCFTR\#1 в клетках [22]. Мы испробовали несколько способов усилить экспрессию этой направляющей РНК: добавили в плазмиду дополнительную кассету (промотор + sgCFTR\#1), соединили sgCFTR\#1 с более активной sgGFP, использовали гибридный промотор U6-tRNAgln, однако ни один из способов не повысил эффективность работы sgCFTR\#1.

Ранее было показано, что для инициации транскрипции c промотора U6 необходимо наличие на 5'-конце направляющей PHK нуклеотидов G или GG [28], поэтому мы либо укоротили имеющиеся sgRNA до первой $\mathrm{G}$, либо добавили их вместо начальных нуклеотидов, чтобы повысить экспрессию и, соответственно, активность sgRNA. Однако такой подход также не привел к ожидаемым результатам.

Исходя из того, что изначально у направляющих РНК был одинаковый промотор, а именно U6, можно предположить, что транскрипция обеих sgRNA должна быть одинаковой.
Разный уровень экспрессии может быть обусловлен более быстрой деградацией sgCFTR\#1 по сравнению с sgGFP. Taк, в исследовании скрининга большого числа направляющих PHK [29] показано, что sgRNA, имеющие в своем составе G-богатые участки (более 8 оснований), более стабильны из-за формирования G-квадруплексов. Но, как и в случае экспериментов по усилению экспрессии, G-квадруплекс не увеличил активность sgCFTR\#1 [22].

В ряде работ сообщается, что нуклеаза Fokl более стабильно работает при $30{ }^{\circ} \mathrm{C}[14,30]$. Мы предположили, что для нуклеазы Cas9 низкая температура культивирования трансфицированных клеток также може быть эфективной, однако такой подход не привел к повышению активности редактирования [22].

Проведенные in vitro эксперименты показывают, что до 41\% направляющих РНК не активны в отношении таргетного локуса [31]. В качестве основной причины рассматривается нуклеотидный состав sgRNA: например, Т- и ТТ-богатые последовательности снижают эффективность [32], присутствие разных нуклеотидов в определенных позициях направляющей РНК также достоверно ассоциировано с разной активностью sgRNA [31]. Кроме того, могут играть роль и вторичные структуры, образуемые направляющими PHK [31]. Если низкая эффективность работы sgRNA связана с ее последовательностью, то единственным решением в таком случае является подбор новой sgRNA.

\section{ВЫВОДЫ}

По результатам редактирования локуса CFTR в клеточной культуре НЕК293Т, наиболее эффективна комбинация из SaCas9 и sgRNA, подобранная на мутацию F508del (редактирование составило 29\%). SgCFTR\#1, подобранная

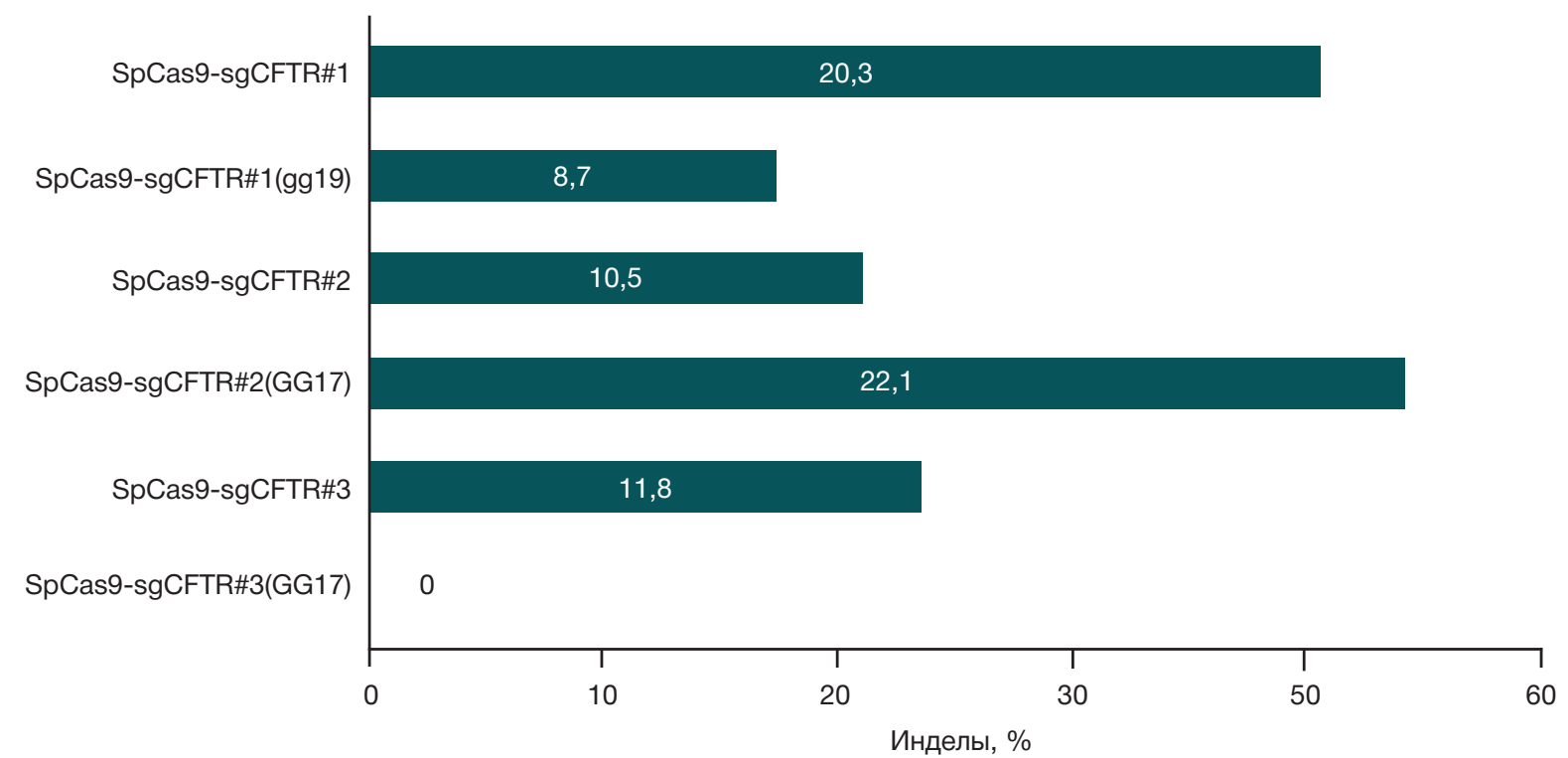

Рис. 6. Сравнение эффективности редактирования CFTR в культуре НЕК293Т с использованием модифицированных sgRNA. Данные представлены как среднее значение

Таблица. Сравнение эффективности редактирования гена CFTR при разных условиях культивирования транссрицированных клеток НЕК293Т

\begin{tabular}{|l|l|l|}
\hline \multicolumn{1}{|c|}{ Условия культивирования } & \multicolumn{1}{|c|}{ Трансфицированные плазмиды } & \multicolumn{1}{|c|}{ Инделы (среднее), \% } \\
\hline \multirow{2}{*}{72 ч при $37^{\circ} \mathrm{C}$} & SpCas9-sgCFTR\#1+ pGEM-TA-CFTR & 17,6 \\
\cline { 2 - 4 } & pGEM-TA-CFTR & 0 \\
\hline \multirow{2}{*}{24 4 при $37^{\circ} \mathrm{C}, 48$ ч при $30^{\circ} \mathrm{C}$} & SpCas9-sgCFTR\#1+ pGEM-TA-CFTR & 10,9 \\
\cline { 2 - 4 } & pGEM-TA-CFTR & 0 \\
\hline
\end{tabular}


на мутацию F508del, в комбинации в двумя разными SpCas9 показала наименьшую эффективность: 13,8\% - с Cas9(1.1) и 8\% - c Cas9(HF4), что связано с ее низкой экспрессией. Предпринятые попытки увеличить экспрессию sgCFTR\#1 путем добавления еще одной экспрессирующей кассеть в плазмиду, соединения этой sgRNA с активной sgGFP и использования гибридного промотора, существенного повышения эффективности работы sgCFTR\#1 не дали. Попытки стабилизировать sgCFTR\#1 путем добавления в ее последовательность G-квадруплекса, укорочения и добавления GG в 5'-область также не принесли результатов. Культивирование редактируемых клеток при более низкой температуре не привело к повышению эффективности редактирования. Таким образом, низкая эффективность работы sgCFTR\#1, вероятно, является следствием ее нуклеотидной последовательности, и необходимо использовать другие направляющие РНК, либо другие Cas9, например, SaCas9, с другими PAMпоследовательностями, расширяющими возможности подбора направляющих PHK на мутацию F508del.

\section{Литература}

1. Красовский С. А., Черняк А. В., Воронкова А. Ю., Амелина Е. Л., Каширская Н. Ю., Кондратьева Е. И. и др. (редакторы) Регистр больных муковисцидозом в Российской Федерации. 2016 год. М.: ИД «МЕДПРАКТИКА-М», 2018, 64 с.

2. Burney TJ, Davies JC. Gene therapy for the treatment of cystic fibrosis. Appl Clin Genet. 2012 May 29; 5: 29-36. DOI: 10.2147/ TACG.S8873.

3. Amaral MD. Novel personalized therapies for cystic fibrosis: treating the basic defect in all patients. J Intern Med. 2015; 277: 55-166.

4. Cohen-Cymberknoh M, Shoseyov D, Kerem E. Managing cystic fibrosis: strategies that increase life expectancy and improve quality of life. Am J Respir Crit Care Med. 2011 Jun 1; 183 (11): 1463-71. DOI: 10.1164/rccm.201009-1478Cl.

5. Whiting P, Al M, Burgers L, Westwood M, Ryder S, Hoogendoorn M, et al. Ivacaftor for the treatment of patients with cystic fibrosis and the G551D mutation: a systematic review and cost-effectiveness analysis. Health Technol Assess. 2014 Mar; 18 (18): 1-106.

6. Mayer M. Lumacaftor-ivacaftor (Orkambi) for cystic fibrosis: behind the 'breakthrough'. Evid Based Med. 2016 Jun; 21 (3): 83-6.

7. Cholon DM, Esther CR Jr, Gentzsch M. Efficacy of lumacaftorivacaftor for the treatment of cystic fibrosis patients homozygous for the F508del-CFTR mutation. Expert Rev Precis Med Drug Dev. 2016; 1 (3): 235-43.

8. Zhang F, Wen Y, Guo X. CRISPR/Cas9 for genome editing: progress, implications and challenges. Hum Mol Genet. 2014 Sep 15; 23 (R1): R40-6.

9. Смирнихина С. А., Лавров А. В. Генная терапия наследственных заболеваний с помощью технологии CRISPR/Cas9 in vivo. Медицинская генетика. 2016; 15 (9): 3-11.

10. Hockemeyer D, Jaenisch R. Induced pluripotent stem cells meet genome editing. Cell Stem Cell. 2016 May 5; 18 (5): 573-86.

11. Maeder ML, Gersbach CA. Genome-editing Technologies for Gene and Cell Therapy. Mol Ther. 2016 Mar; 24 (3): 430-46.

12. Prakash V, Moore M, Yáñez-Muñoz RJ. Current Progress in Therapeutic Gene Editing for Monogenic Diseases. Mol Ther. 2016 Mar; 24 (3): 465-74.

13. Firth AL, Menon T, Parker GS, Qualls SJ, Lewis BM, Ke E, et al. Functional Gene Correction for Cystic Fibrosis in Lung Epithelia Cells Generated from Patient iPSCs. Cell Rep. 2015 Sep 1; 12 (9): 1385-90.

14. Lee CM, Flynn R, Hollywood JA, Scallan MF, Harrison PT. Correction of the $\triangle \mathrm{F} 508$ Mutation in the Cystic Fibrosis Transmembrane Conductance Regulator Gene by Zinc-Finger Nuclease Homology-Directed Repair. BioResearch Open Access. 2012; 1 (3): 99-108.

15. Suzuki S, Sargent RG, Illek B, Fischer H, Esmaeili-Shandiz A Yezzi MJ, et al. TALENs Facilitate Single-step Seamless SDF Correction of F508del CFTR in Airway Epithelial Submucosal Gland Cell-derived CF-iPSCs. Mol Ther Nucleic Acids. 2016 Jan 5; 5: e273.

16. Hollywood JA, Lee CM, Scallan MF, Harrison PT. Analysis of gene repair tracts from Cas9/gRNA double-stranded breaks in the human CFTR gene. Sci Rep. 2016 Aug 25; 6: 32230

17. Crane AM, Kramer P, Bui JH, Chung WJ, Li XS, GonzalezGaray ML, et al. Targeted correction and restored function of the CFTR gene in cystic fibrosis induced pluripotent stem cells. Stem
Cell Reports. 2015 Apr 14; 4 (4): 569-77.

18. Bednarski C, Tomczak K, Vom Hövel B, Weber WM, Cathomen T. Targeted Integration of a Super-Exon into the CFTR Locus Leads to Functional Correction of a Cystic Fibrosis Cell Line Model. PLoS One. 2016 Aug 15; 11 (8): e0161072.

19. Camarasa MV, Gálvez VM. Robust method for TALEN-edited correction of pF508del in patient-specific induced pluripotent stem cells. Stem Cell Res Ther. 2016 Feb 9; 7: 26.

20. Schwank G, Koo BK, Sasselli V, Dekkers JF, Heo I, Demircan T, et al. Functional repair of CFTR by CRISPR/Cas9 in intestinal stem cell organoids of cystic fibrosis patients. Cell Stem Cell. 2013 Dec 5; 13 (6): 653-58.

21. Смирнихина С. А., Банников А. В., Лавров А. В. Оптимизация условий трансфекции клеточной культуры CFTE290- для разработки редактирования мутации F508del в гене CFTR. Медицинская генетика. 2016; 15 (8): 36-9.

22. Смирнихина С. А., Банников А. В., Анучина А. А., КочергинНикитский К. С., Адильгереева Э. П., Лавров А. В. Факторы, влияющие на эфффективность CRISPR/Cas9 для коррекции мутации F508del при муковисцидозе. Медицинская генетика, 2017; 16 (11): 32-7.

23. Slaymaker IM, Gao L, Zetsche B, Scott DA, Yan WX, Zhang F. Rationally engineered Cas9 nucleases with improved specificity. Science. 2016 Jan 1; 351 (6268): 84-8.

24. Kleinstiver BP, Pattanayak V, Prew MS, Tsai SQ, Nguyen NT, Zheng Z, et al. High-fidelity CRISPR-Cas9 nucleases with no detectable genome-wide off-target effects. Nature. 2016; 529: 490-95.

25. Ran FA, Cong L, Yan WX, Scott DA, Gootenberg JS, Kriz AJ, et al. In vivo genome editing using Staphylococcus aureus Cas9. Nature. 2015 Apr 9; 520 (7546): 186-91.

26. Schwartz CM, Hussain MS, Blenner M, Wheeldon I. Synthetic RNA Polymerase III Promoters Facilitate High-Efficiency CRISPRCas9-Mediated Genome Editing in Yarrowia lipolytica. ACS Synth Biol. 2016 Apr 15; 5 (4): 356-9. DOI: 10.1021/acssynbio.5b00162.

27. Mefferd AL, Kornepati AV, Bogerd HP, Kennedy EM, Cullen BR. Expression of CRISPR/Cas single guide RNAs using small tRNA promoters. RNA. 2015 Sep; 21 (9): 1683-9. DOI: 10.1261/ rna.051631.115.

28. Sander JD, Joung JK. CRISPR-Cas systems for editing, regulating and targeting genomes. Nat Biotechnol. 2014 Apr; 32 (4): 34755. DOI: 10.1038/nbt.2842.

29. Moreno-Mateos MA, Vejnar CE, Beaudoin JD, Fernandez JP, Mis EK, Khokha MK, et al. CRISPRscan: designing highly efficient sgRNAs for CRISPR-Cas9 targeting in vivo. Nat Methods. 2015 Oct: 12 (10): 982-88.

30. Doyon Y, Choi VM, Xia DF, Vo TD, Gregory PD, Holmes MC. Transient cold shock enhances zinc-finger nuclease-mediated gene disruption. Nat Methods. 2010 Jun; 7 (6): 459-60.

31. Liu X, Homma A, Sayadi J, Yang S, Ohashi J, Takumi T. Sequence features associated with the cleavage efficiency of CRISPR/ Cas9 system. Sci Rep. 2016 Jan 27; 6: 19675. DOI: 10.1038/ srep19675

32. Kuan PF, Powers S, He S, Li K, Zhao X, Huang B. A systematic evaluation of nucleotide properties for CRISPR sgRNA design. BMC Bioinformatics. 2017 Jun 6; 18 (1): 297. DOl: 10.1186/ s12859-017-1697-6. 
1. Krasovsky SA, Chernyak AV, Voronkov AY, Amelina EL, Kashirskaya NY, Kondratieva El, i dr. Register of cystic fibrosis patiens in Russian Federation. 2016. Moskva: «MEDPRAKTIKA-M», 2018, 64 s.

2. Burney TJ, Davies JC. Gene therapy for the treatment of cystic fibrosis. Appl Clin Genet. 2012 May 29; 5: 29-36. DOI: 10.2147/ TACG.S8873.

3. Amaral MD. Novel personalized therapies for cystic fibrosis: treating the basic defect in all patients. J Intern Med. 2015; 277: 55-166.

4. Cohen-Cymberknoh M, Shoseyov D, Kerem E. Managing cystic fibrosis: strategies that increase life expectancy and improve quality of life. Am J Respir Crit Care Med. 2011 Jun 1; 183 (11): 1463-71. DOl: 10.1164/rccm.201009-1478Cl.

5. Whiting P, Al M, Burgers L, Westwood M, Ryder S, Hoogendoorn $M$, et al. Ivacaftor for the treatment of patients with cystic fibrosis and the G551D mutation: a systematic review and costeffectiveness analysis. Health Technol Assess. 2014 Mar; 18 (18): 1-106.

6. Mayer M. Lumacaftor-ivacaftor (Orkambi) for cystic fibrosis: behind the 'breakthrough'. Evid Based Med. 2016 Jun; 21 (3): 83-6.

7. Cholon DM, Esther CR Jr, Gentzsch M. Efficacy of lumacaftorivacaftor for the treatment of cystic fibrosis patients homozygous for the F508del-CFTR mutation. Expert Rev Precis Med Drug Dev. 2016; 1 (3): 235-43.

8. Zhang F, Wen Y, Guo X. CRISPR/Cas9 for genome editing: progress, implications and challenges. Hum Mol Genet. 2014 Sep 15; 23 (R1): R40-6.

9. Smirnikhina SA, Lavrov AV. Gennaja terapija nasledstvennyh zabolevanij s pomoshh'ju tehnologii CRISPR/Cas9 in vivo. Medicinskaja genetika. 2016; 15 (9): 3-11.

10. Hockemeyer D, Jaenisch R. Induced pluripotent stem cells meet genome editing. Cell Stem Cell. 2016 May 5; 18 (5): 573-86.

11. Maeder ML, Gersbach CA. Genome-editing Technologies for Gene and Cell Therapy. Mol Ther. 2016 Mar; 24 (3): 430-46.

12. Prakash V, Moore M, Yáñez-Muñoz RJ. Current Progress in Therapeutic Gene Editing for Monogenic Diseases. Mol Ther 2016 Mar; 24 (3): 465-74.

13. Firth AL, Menon T, Parker GS, Qualls SJ, Lewis BM, Ke E, et al Functional Gene Correction for Cystic Fibrosis in Lung Epithelial Cells Generated from Patient iPSCs. Cell Rep. 2015 Sep 1; 12 (9) 1385-90.

14. Lee CM, Flynn R, Hollywood JA, Scallan MF, Harrison PT. Correction of the $\triangle \mathrm{F} 508$ Mutation in the Cystic Fibrosis Transmembrane Conductance Regulator Gene by Zinc-Finger Nuclease Homology-Directed Repair. BioResearch Open Access. 2012; 1 (3): 99-108.

15. Suzuki S, Sargent RG, Illek B, Fischer H, Esmaeili-Shandiz A Yezzi MJ, et al. TALENs Facilitate Single-step Seamless SDF Correction of F508del CFTR in Airway Epithelial Submucosal Gland Cell-derived CF-iPSCs. Mol Ther Nucleic Acids. 2016 Jan 5; 5: e273.

16. Hollywood JA, Lee CM, Scallan MF, Harrison PT. Analysis of gene repair tracts from Cas9/gRNA double-stranded breaks in the human CFTR gene. Sci Rep. 2016 Aug 25; 6: 32230

17. Crane AM, Kramer P, Bui JH, Chung WJ, Li XS, Gonzalez-Garay $\mathrm{ML}$, et al. Targeted correction and restored function of the CFTR gene in cystic fibrosis induced pluripotent stem cells. Stem Cell Reports. 2015 Apr 14; 4 (4): 569-77.

18. Bednarski C, Tomczak K, Vom Hövel B, Weber WM, Cathomen T. Targeted Integration of a Super-Exon into the CFTR Locus Leads to Functional Correction of a Cystic Fibrosis Cell Line Model. PLoS One. 2016 Aug 15; 11 (8): e0161072.

19. Camarasa MV, Gálvez VM. Robust method for TALEN-edited correction of pF508del in patient-specific induced pluripotent stem cells. Stem Cell Res Ther. 2016 Feb 9; 7: 26

20. Schwank G, Koo BK, Sasselli V, Dekkers JF, Heo I, Demircan T, et al. Functional repair of CFTR by CRISPR/Cas9 in intestinal stem cell organoids of cystic fibrosis patients. Cell Stem Cell. 2013 Dec 5; 13 (6): 653-58.

21. Smirnikhina SA, Bannikov AV, Lavrov AV. Optimizacija uslovij transfekcii kletochnoj kul'tury CFTE29o- dlja razrabotki redaktirovanija mutacii F508del $v$ gene CFTR. Medicinskaja genetika. 2016; 15 (8): 36-9.

22. Smirnikhina SA, Bannikov AV, Anuchina AA, Kochergin-Nikitsky KS, Adilgereeva EP, Lavrov AV. Faktory, vlijajushhie na jeffektivnost' CRISPR/Cas9 dlja korrekcii mutacii F508del pri mukoviscidoze. Medicinskaja genetika, 2017; 16 (11) s. 32-7.

23. Slaymaker IM, Gao L, Zetsche B, Scott DA, Yan WX, Zhang F. Rationally engineered Cas9 nucleases with improved specificity. Science. 2016 Jan 1; 351 (6268): 84-8.

24. Kleinstiver BP, Pattanayak V, Prew MS, Tsai SQ, Nguyen NT, Zheng Z, et al. High-fidelity CRISPR-Cas9 nucleases with no detectable genome-wide off-target effects. Nature. 2016; 529: 490-95.

25. Ran FA, Cong L, Yan WX, Scott DA, Gootenberg JS, Kriz AJ, et al. In vivo genome editing using Staphylococcus aureus Cas9. Nature. 2015 Apr 9; 520 (7546): 186-91.

26. Schwartz CM, Hussain MS, Blenner M, Wheeldon I. Synthetic RNA Polymerase III Promoters Facilitate High-Efficiency CRISPRCas9-Mediated Genome Editing in Yarrowia lipolytica. ACS Synth Biol. 2016 Apr 15; 5 (4): 356-9. DOI: 10.1021/acssynbio.5b00162.

27. Mefferd AL, Kornepati AV, Bogerd HP, Kennedy EM, Cullen BR. Expression of CRISPR/Cas single guide RNAs using small tRNA promoters. RNA. 2015 Sep; 21 (9): 1683-9. DOI: 10.1261/ rna.051631.115.

28. Sander JD, Joung JK. CRISPR-Cas systems for editing, regulating and targeting genomes. Nat Biotechnol. 2014 Apr; 32 (4): 34755. DOl: $10.1038 / \mathrm{nbt} .2842$.

29. Moreno-Mateos MA, Vejnar CE, Beaudoin JD, Fernandez JP, Mis EK, Khokha MK, et al. CRISPRscan: designing highly efficient sgRNAs for CRISPR-Cas9 targeting in vivo. Nat Methods. 2015 Oct; 12 (10): 982-88.

30. Doyon Y, Choi VM, Xia DF, Vo TD, Gregory PD, Holmes MC Transient cold shock enhances zinc-finger nuclease-mediated gene disruption. Nat Methods. 2010 Jun; 7 (6): 459-60.

31. Liu X, Homma A, Sayadi J, Yang S, Ohashi J, Takumi T. Sequence features associated with the cleavage efficiency of CRISPR/ Cas9 system. Sci Rep. 2016 Jan 27; 6: 19675. DOI: 10.1038/ srep19675.

32. Kuan PF, Powers S, He S, Li K, Zhao X, Huang B. A systematic evaluation of nucleotide properties for CRISPR sgRNA design. BMC Bioinformatics. 2017 Jun 6; 18 (1): 297. DOl: 10.1186/ s12859-017-1697-6. 